\title{
INFLUÊNCIA DO MÉTODO HANDS-OFF NA INTEGRIDADE PERINEAL, PROBLEMAS PUERPERAIS E NEONATAIS
}

\author{
Maria Alice Leony de Paiva ${ }^{1}$; Ariane Cedraz Morais ${ }^{2}$, Luciano Marques dos Santos ${ }^{3}$ \\ 1. Bolsista PROBIC, Graduanda em Enfermagem, Universidade Estadual de Feira de Santana, e-mail: \\ alice_pitombo@hotmail.com \\ 2. Orientadora, Departamento de Saúde, Universidade Estadual de Feira de Santana, e-mail: \\ enfarianecedraz@hotmail.com \\ 3. Orientador, Departamento de Saúde, Universidade Estadual de Feira de Santana, Pesquisador do \\ NUDES, e-mail: lucmarxenfo@yahoo.com.br
}

PALAVRAS-CHAVE: Enfermagem obstétrica; períneo; período pós-parto.

\section{INTRODUÇÃO}

Em decorrência do trauma perineal no parto vaginal, como a laceração perineal e episiotomia, as mulheres ficam sujeitas a diversas morbidades como dor perineal, sangramento aumentado, disfunções intestinais, urinárias e piora na função sexual em relação àquelas que não sofreram esse agravo durante o parto' ${ }^{1}$.

Diante disso, surgiram os métodos de proteção perineal que são empregados para reduzir o risco de trauma perineal. Esses métodos são: a posição materna no período expulsivo do parto do tipo não litotômica, massagem perineal, compressas quentes, método hands-on e hands-off 2

Um estudo randomizado controlado, realizado no Iran com 600 nulíparas divididas igualmente entre dois grupos hands-on e hands-off, os traumas de terceiro grau foram superiores no grupo hands-on com 2,7\% em comparação com $0,3 \%(\mathrm{p}=0,1)$ no hands-off. A episiotomia também foi superior no grupo hands-on com 12,7\% (38 mulheres) e 5,7\% (17 mulheres) no hands-off ${ }^{3}$.

Um outro estudo randomizado controlado também mostrou que no método hands-on a ocorrência de lacerações perineais de terceiro grau $(2,7 \%)$ e episiotomia $(17,9 \%)$ foi maior quando comparado ao hands-off com $0,9 \%$ e $10,1 \%$ respectivamente ${ }^{4}$. Por isso, uma postura expectante (hands-off) pode ser preferível a uma postura ativa, ou seja, hands-on ${ }^{3}$. Poucos ensaios clínicos randomizados sobre tais método incluem número suficiente de parturientes e com boa qualidade metodológica.

Por isso, sugere-se que o método hands-on não deve ser usado rotineiramente porque interfere no curso do mecanismo do parto, e que a decisão de utilizar esses métodos seja tomada pela mulher, respeitando sua autonomia neste processo ${ }^{5}$.

O método hands-off no parto vaginal oferece mais vantagens para a saúde materna, diante da menor taxa de episiotomia e laceração de terceiro grau, sendo por isso um método mais seguro. Porém, mais estudos são necessários para avaliar a segurança e eficácia desses métodos, bem como os fatores que contribuem para a ocorrência do trauma perineal durante o parto $^{3}$.

Diante desse contexto, este estudo tem como objeto de investigação a influência do método hands-off durante o parto vaginal na ocorrência de traumas e suturas perineais e problemas puerperais. Ao realizar o levantamento do estado da arte foram encontrados 17 artigos relacionados ao objeto em estudo. Percebeu-se que poucos estudos brasileiros abordam a utilização dos métodos hands-on e hands-off durante o processo parturitivo.

Esse estudo teve como objetivo geral verificar a influência do método hands-off na ocorrência de trauma e sutura perineal, e problemas locais em mulheres com parto vaginal em uma maternidade pública de Feira de Santana na Bahia, e objetivos específicos: avaliar a taxa de ocorrência e local do trauma perineal conforme o tipo de método utilizado no parto vaginal, 
para recepção do recém-nascido; analisar a associação entre os métodos hands-on e hands-off com a ocorrência e local de trauma perineal no pós-parto imediato; verificar a associação entre o método hands-on e hands-off com a necessidade de sutura perineal e verificar a associação entre os métodos hands-on e hands-off com a ocorrência de problemas puerperais.

\section{MÉTODOS}

Trata-se de um estudo do tipo caso-controle, caracterizado como observacional e analítico vinculado ao projeto de pesquisa intitulado "Influência da postura e método hands-off no parto vaginal na integridade perineal e comorbidades maternas e neonatais no puerpério imediato", realizado no Hospital Inácia Pinto dos Santos (HIPS) no município de Feira de Santana, na Bahia, aprovado sob o número do parecer 1.668.328.

A população deste estudo foi composta por todos os prontuários de puérperas submetidas ao parto vaginal na unidade em estudo. A amostra deste estudo foi do tipo por conveniência e calculada de forma estatística, totalizando 376 prontuários.

Inicialmente, foi realizada a leitura do livro de ocorrência do centro obstétrico do HIPS visando identificar as mulheres que tiveram partos por via vaginal no período de 2014 a 2016. Nesse livro de registro, para cada parto com método hands-off foi selecionado o parto com método hands-on subsequente. Foram identificados 188 partos vaginais realizados por médicos que fizeram parte do grupo controle, já que estes profissionais utilizam o método hands-on e 188 partos vaginais realizados por enfermeiros que utilizam o método hands-off em sua prática, e que compuseram o grupo caso. As mulheres do grupo controle foram pareadas com as mulheres do grupo caso individualmente, isto é, um caso para um controle, tendo como critérios de pareamento a idade e paridade. A seguir, foram consultados os prontuários das mulheres selecionadas para o estudo no Serviço de Arquivamento Médico e Estatístico do HIPS, para esta etapa do projeto foi elaborado um formulário.

Os dados coletados foram digitados, tratados e analisados por meio do pacote estatístico Statistical Package for the Social Sciences (SPSS), versão 22.0.Para determinar a associação entre as variáveis de exposição e desfecho, foi empregado o McNemar e cálculo do odds ratio (OR), com nível de significância de 5\% (p<0,05) e intervalos de confiança de 95\%. Este estudo respeitou a Resolução 466/12 do Conselho Nacional de Saúde. Por não envolver diretamente a participação de seres humanos não foi necessária a utilização do TCLE nesse estudo.

\section{RESULTADOS}

Foram estudadas 376 mulheres, das quais $51,9 \%$ tinham idade entre 21 e 30 anos (média 24,8 anos $\pm 6,4$ ), $51,1 \%$ eram nulíparas (média de 1 parto $\pm 1,2$ ), 40,7\% receberam ocitocina intravenosa, $54 \%$ dos partos foram na postura litotômica e $36,7 \%$ no período matutino.

Tabela 1. Associação entre método de desprendimento cefálico com a integridade perineal, tipo de trauma, grau da laceração, local do trauma, ocorrência de sutura, tipo de sutura e de fio utilizado, e postura adotada durante o parto de mulheres que tiveram parto vaginal em uma instituição pública, Feira de Santana (Bahia), Julho/2016-outubro/2016

\begin{tabular}{|c|c|c|c|c|c|c|}
\hline \multirow[b]{2}{*}{$\begin{array}{l}\text { Integridade Perineal } \\
\text { Períneo não-íntegro }\end{array}$} & & Hands-off & Hands-on & \multirow[b]{2}{*}{$\begin{array}{l}\text { OR } \\
4,6\end{array}$} & \multirow[b]{2}{*}{$\begin{array}{c}\text { IC } \\
3,2-6,7\end{array}$} & \multirow[b]{2}{*}{$\begin{array}{c}\mathrm{p} \text {-valor } \\
0,000\end{array}$} \\
\hline & $N(\%)$ & $154(81,9)$ & $158(84)$ & & & \\
\hline $\begin{array}{l}\text { Períneo íntegro } \\
\text { Trauma perineal }\end{array}$ & $\mathrm{N}(\%)$ & $34(18,1)$ & $30(16)$ & & & \\
\hline Episiotomia & $\begin{array}{l}\text { Sim N }(\%) \\
\text { Não N (\%) }\end{array}$ & $\begin{array}{c}5(2,7) \\
183(97,3)\end{array}$ & $\begin{array}{l}58(30,9) \\
130(69,1)\end{array}$ & 0,4 & $0,24-0,43$ & 0,000 \\
\hline Laceração perineal & $\begin{array}{l}\text { Sim N (\%) } \\
\text { Não N (\%) }\end{array}$ & $\begin{array}{c}149(79,3) \\
39(20,7)\end{array}$ & $\begin{array}{l}97(51,6) \\
91(48,4)\end{array}$ & 2,4 & $1,7-3,6$ & 0,000 \\
\hline
\end{tabular}




\begin{tabular}{|c|c|c|c|c|c|c|}
\hline \multicolumn{7}{|l|}{ Grau da laceração } \\
\hline \multirow{2}{*}{ Laceração de $1^{\circ}$ grau } & $\operatorname{Sim} N(\%)$ & $115(77,2)$ & $41(62,1)$ & 1,20 & $0,76-1,9$ & 0,4189 \\
\hline & Não N (\%) & $34(22,8)$ & $25(37,9)$ & & & \\
\hline \multirow[t]{2}{*}{ Laceração de $2^{\circ}$ grau } & $\operatorname{Sim} N(\%)$ & $47(31,5)$ & $28(42,4)$ & 0,27 & $0,18-0,41$ & 0,000 \\
\hline & Não N (\%) & $102(68,5)$ & $38(57,6)$ & & & \\
\hline \multirow[t]{2}{*}{ Laceração de $3^{\circ}$ grau } & $\operatorname{Sim} N(\%)$ & $10(6,7)$ & $3(4,5)$ & 0,02 & $0,006-0,067$ & 0,000 \\
\hline & Não N (\%) & $139(93,3)$ & $63(95,5)$ & & & \\
\hline \multicolumn{7}{|l|}{ Local do trauma } \\
\hline \multirow[t]{2}{*}{ Região anterior } & $\operatorname{Sim} N(\%)$ & $110(71,9)$ & $39(32)$ & 0,9 & $0,58-1,39$ & 0,658 \\
\hline & Não N (\%) & $43(28,1)$ & $83(68)$ & & & \\
\hline \multirow[t]{2}{*}{ Região posterior } & $\operatorname{Sim} N(\%)$ & $28(18,3)$ & $75(61,5)$ & 0,6 & $0,45-0,79$ & 0,000 \\
\hline & Não N (\%) & $125(81,7)$ & $47(38,5)$ & & & \\
\hline \multirow{2}{*}{$\begin{array}{l}\text { Região anterior e } \\
\text { posterior }\end{array}$} & $\operatorname{Sim} N(\%)$ & $18(11,8)$ & $7(5,7)$ & 0,05 & $0,02-0,11$ & 0,000 \\
\hline & Não N (\%) & $135(88,2)$ & $115(94,3)$ & & & \\
\hline \multirow{2}{*}{ Parede vaginal } & $\operatorname{Sim} N(\%)$ & $8(5,2)$ & $3(2,5)$ & 0,02 & $0,006-0,06$ & 0,000 \\
\hline & Não N (\%) & $145(94,8)$ & $119(97,5)$ & & & \\
\hline \multicolumn{7}{|l|}{$\begin{array}{l}\text { Ocorrência de sutura } \\
\qquad(n=308)\end{array}$} \\
\hline Sem sutura & $\mathrm{N}(\%)$ & $177(63,9)$ & $48(25,1)$ & 1,48 & $3,4-0,67$ & 0,000 \\
\hline Com sutura & $\mathrm{N}(\%)$ & $100(36,1)$ & $143(74,9)$ & & & \\
\hline \multicolumn{7}{|l|}{ Tipo de sutura $(n=74)$} \\
\hline Separada & $\mathrm{N}(\%)$ & $3(4,5)$ & $26(52)$ & 0,4 & $2,5-0,64$ & 0,000 \\
\hline Contínua & $\mathrm{N}(\%)$ & $64(95,5)$ & $24(48)$ & & & \\
\hline \multicolumn{7}{|l|}{$\begin{array}{l}\text { Tipo de fio utilizado } \\
\qquad(\mathrm{n}=163)\end{array}$} \\
\hline Simples 2.0 & $\mathrm{~N}(\%)$ & $5(5)$ & $2(1,7)$ & 0,02 & $0,05-0,08$ & 0,000 \\
\hline Cromado 2.0 & $\mathrm{~N}(\%)$ & $95(95)$ & $116(98,3)$ & & & \\
\hline \multicolumn{7}{|l|}{$\begin{array}{l}\text { Postura adotada durante } \\
\text { o parto }\end{array}$} \\
\hline Litotômica & $\mathrm{N}(\%)$ & $18(9,6)$ & $185(98,4)$ & 1,088 & $0,8-1,3$ & 0,42 \\
\hline Não-litotômica & $\mathrm{N}(\%)$ & $170(90,4)$ & $3(1,6)$ & & & \\
\hline
\end{tabular}

FONTE: Banco de dados do Projeto de Pesquisa "Influência da postura e método hands-off no parto vaginal na integridade perineal e comorbidades maternas e neonatais no puerpério imediato", Feira de Santana, 2016.

Tabela 2. Associação entre método de desprendimento cefálico com problemas puerperais, problemas neonatais e condutas adotadas imediatamente após o parto vaginal de mulheres em uma instituição pública, Feira de Santana (Bahia), Julho/2016-outubro/2016

\section{Problemas puerperais}

Edema $(n=227)$

Hemorragia no puerpério imediato $(\mathrm{n}=225)$

Dor perineal $(\mathrm{n}=225)$

\section{Problemas neonatais}

Apgar $1^{\circ}$ minuto $(\mathrm{n}=372)$
Hands-off Hands-on

$\begin{array}{lccccc}\text { Sim } & 17(14) & 20(18,9) & 0,19 & 0,11-0,31 & 0,000 \\ \text { Não } & 104(86) & 86(81,1) & & & \\ \text { Sim } & 3(2,5) & 1(1) & 0,008 & 0,001- & 0,000 \\ & & & & 0,06 & \end{array}$

$\begin{array}{lccccc}\text { Não } & 117(97,5) & 104(99) & & & \\ \text { Sim } & 16(13,4) & 21(19,8) & 0,20 & 0,12-0,32 & 0,000 \\ & & & & & \\ \text { Não } & 103(86,6) & 85(80,2) & & & \end{array}$

\begin{tabular}{cccccc}
$\begin{array}{c}\text { Menor ou igual a } \\
7 \mathrm{~N}(\%)\end{array}$ & $10(5,3)$ & $18(9,7)$ & 0,1 & $0,06-0,16$ & 0,000 \\
Maior que 7 & $177(94,7)$ & $167(90,3)$ & & & \\
\hline
\end{tabular}




\begin{tabular}{|c|c|c|c|c|c|c|}
\hline \multirow{3}{*}{ Apgar $5^{\circ}$ minuto $(n=361)$} & $\mathrm{N}(\%)$ & & & & & \\
\hline & Menor ou igual a & $2(1,1)$ & $1(0,6)$ & 0,005 & $0,0-0,04$ & 0,000 \\
\hline & $\begin{array}{c}\text { Maior que } 7 \\
\mathrm{~N}(\%)\end{array}$ & $178(98,9)$ & $180(99,4)$ & & & \\
\hline \multirow{2}{*}{$\begin{array}{l}\text { Reanimação neonatal } \\
(\mathrm{n}=375)\end{array}$} & Sim & $8(4,3)$ & $20(10,6)$ & 0,1 & $0,07-0,17$ & 0,000 \\
\hline & Não & $179(95,7)$ & $168(89,4)$ & & & \\
\hline \multirow[t]{2}{*}{$\begin{array}{l}\text { Conduta utilizada na } \\
\text { reanimação }\end{array}$} & $\begin{array}{l}\text { Só cuidados } \\
\text { iniciais } \\
\mathrm{N}(\%)\end{array}$ & $5(62,5)$ & $14(70)$ & 4,6 & $\begin{array}{l}0,34- \\
16,24\end{array}$ & 0,007 \\
\hline & $\begin{array}{l}\text { Cuidados iniciais } \\
\text { e VPP N(\%) }\end{array}$ & $3(37,5)$ & $6(30)$ & & & \\
\hline \multicolumn{7}{|l|}{$\begin{array}{c}\text { Condutas adotadas } \\
\text { imediatamente após o } \\
\text { parto }\end{array}$} \\
\hline \multirow{2}{*}{$\begin{array}{l}\text { Aleitamento materno } \\
\qquad(\mathrm{n}=375)\end{array}$} & Sim & $163(91,6)$ & $26(17,3)$ & 1,73 & $0,9-3,2$ & 0,085 \\
\hline & Não & $15(8,4)$ & $124(82,7)$ & & & \\
\hline $\begin{array}{l}\text { A Contato pele a pele } \\
(\mathrm{n}=375)\end{array}$ & Sim & $173(97,2)$ & $90(60)$ & 14 & $7,3-44,2$ & 0,000 \\
\hline \multirow{3}{*}{$\begin{array}{c}\text { Clampeamento do cordão } \\
\text { umbilical } \\
(\mathrm{n}=308)\end{array}$} & Não & $7(4,5)$ & $64(41,8)$ & & & \\
\hline & Imediato & $12(6,4)$ & $102(54,3)$ & 0,5 & $0,45-0,73$ & 0,000 \\
\hline & Tardio & $176(93,6)$ & $86(45,77)$ & & & \\
\hline
\end{tabular}

FONTE: Banco de dados do Projeto de Pesquisa "Influência da postura e método hands-off no parto vaginal na integridade perineal e comorbidades maternas e neonatais no puerpério imediato", Feira de Santana, 2016.

\section{CONCLUSÃO}

Mulheres assistidas pelo método hands-off tiveram mais chances de apresentarem lacerações perineais, entretanto, a maioria foram lacerações de baixo grau (primeiro grau), que não demandam sutura perineal e são de rápida cicatrização, já que são lesões superficiais que acometem apenas a pele. Já as mulheres assistidas pelo método hands-on tiveram mais chances de serem submetidas à episiotomia, que é um trauma mais severo e profundo que demanda de sutura perineal, e por isso está associada com maior ocorrência de complicações no pós-parto. Diante disso sugere-se que o método hands-off seja mais utilizado na prática clínica pelos profissionais responsáveis pelo parto. Os dados desse estudo podem contribuir com a incipiente produção do conhecimento relacionada ao objeto estudado, estimulando a realização de novas pesquisas sobre a temática. Na prática, pode proporcionar uma maior visibilidade e aplicabilidade do método hands-off por profissionais responsáveis pelo parto, e no âmbito social pode contribuir para uma possível redução de problemas puerperais decorrentes do parto vaginal.

\section{REFERÊNCIAS}

BELEZA, A.C.S et al. 2012. [online]. Mensuração e caracterização da dor após episiotomia e sua relação com a limitação de atividades. Rev bras enferm Brasília 65(2): 264-8.

FRANCISCO, A.A et al. 2011. [online]. Avaliação e tratamento da dor perineal no pós-parto vaginal. Acta paul enferm. 24(1):94-100.

FRANCISCO AA, et al. 2012. [online]. Efecto del dolor perineal em las actividades de mujeres sometidas a episiotomía. Index enferm Granada. 21(3):150-4.

PITANGUI, A.C.R et al. 2009.[online]. Measurement and characteristics of perineal pain in primiparous undergoing episiotomy. Acta paul enferm. 22 (1): 77-82.

SANTOS, L.M; PEREIRA, S.S.C. 2012. [online]. Vivências de mulheres sobre a assistência recebida no processo parturitivo. Physis Rev Saúde Coletiva. 22 (1): 77-97. 